\title{
Optics and optics-based technologies education with the benefit of LabVIEW
}

Yuhong Wan, Tianlong Man, Shiquan Tao

Yuhong Wan, Tianlong Man, Shiquan Tao, "Optics and optics-based technologies education with the benefit of LabVIEW," Proc. SPIE 9793, Education and Training in Optics and Photonics: ETOP 2015, 979310 (8 October 2015); doi: 10.1117/12.2223124

SPIE Event: Education and Training in Optics and Photonics: ETOP 2015, 2015, Bordeaux, France 


\title{
Optics and Optics-based Technologies Education With the Benefit of
}

\author{
LabVIEW \\ Yuhong Wan Tianlong Man Shiquan Tao \\ College of Applied Science, Beijing University of Technology, Beijing, 100124, CHINA \\ *Corresponding author: yhongw@ bjut.edu.cn
}

\begin{abstract}
The details of design and implementation of incoherent digital holographic experiments based on LabVIEW are demonstrated in this work in order to offer a teaching modal by making full use of LabVIEW as an educational tool. Digital incoherent holography enables holograms to be recorded from incoherent light with just a digital camera and spatial light modulator, and three-dimensional properties of the specimen are revealed after the hologram is reconstructed in the computer. The experiment of phase shifting incoherent digital holography is designed and implemented based on the principle of Fresnel incoherent correlation holography. An automatic control application is developed based on LabVIEW, which combines the functions of major experimental hardware control and digital reconstruction of the holograms. The basic functions of the system are completed and a user-friendly interface is provided for easy operation. The students are encouraged and stimulated to learn and practice the basic principle of incoherent digital holography and other related optics-based technologies during the programming of the application and implementation of the system.
\end{abstract}

\section{Introduction}

NI LabVIEW system design software is a graphical programming platform of National instruments. The comprehensive tool for building any experimental control applications in laboratory provided by LabVIEW graphical programming method makes it easier for problem solving, accelerated productivity, and continual innovation. The modularization designed platform provides a more readable program for the application of complex systems. Various kinds of data processing are also possible when combing LabVIEW with any mathematical computational software such as Matlab.

LabVIEW has been widely introduced into the teaching in classroom by visualizing the abstract physical process and making it easier to be understood by the students. In our previous works, various methods are applied to information optics teaching in order to improve the teaching quality [1]. Here LabVIEW is introduced into the teaching for stimulating the interest of students in referring the basic principle and discovering innovation applications. In practical, a research project of programming the control application for incoherent digital holographic experiment using LabVIEW is established and applied in the teaching. The undergraduate students who select the

\footnotetext{
Education and Training in Optics and Photonics: ETOP 2015, edited by Eric Cormier, Laurent Sarger
} Proc. of SPIE Vol. 9793, 979310 • (c) 2015 SPIE, IEEE, OSA, ICO · doi: 10.1117/12.2223124 
course are encouraged and expected to better understanding the basic principles of digital holography such as the diffraction, interference and propagation of light. Furthermore, some advanced scientific research results of us have been introduced for extending the knowledge of the students about the coherence of the light sources.

Spatially incoherent digital holography was proposed to achieve the holographic recording and three-dimensional (3D) reconstruction of the spatially incoherent illuminated or self luminous objects. The basic concept of spatially incoherent digital holography was first proposed by Mertz and Young in astronomical applications [2]. Various investigations have been done during the further developed of the techniques. Among them, Fresnel incoherent correlation holography (FINCH) has been considered to be a promising and efficient method, where the hologram of the spatially incoherent object is recorded basing on the correlation of the intensity distribution of the object and the Fresnel-zone-plate-like point sources' interference pattern [3]. The object is assumed to consist of many point light sources, where any pairs of the points are spatially incoherent. Light emitted from any point is split into two beams using spatial light modulator (SLM). The two beams can interference because they are originated from the same point. The interference pattern is coined as point source hologram here. The hologram of the extended object is the summation of all the point source holograms, and a digital camera is used to capture the hologram. Phase shifting techniques is implemented for eliminating the twin image and zero order in the recorded hologram. SLM is used as beam splitter and phase shifter in the system by displaying the diffraction optical element (DOE). In the system, the DOE is equivalent to a combination of two positive lenses that have different focal lengths, where the constant phase values necessary for the phase shifting are introduced in one of the lenses. A series of holograms with different phase values are captured sequentially, then a complex hologram can be obtained using phase shifting formulas. The hologram is reconstructed to obtain the 3D image of the object by using Fresnel propagation algorithm in the computer. In this paper, a control application is developed based on LabVIEW, which combines the functions of major experimental hardware control and digital reconstruction of the holograms in FINCH. During the programming of the application, the students will be deeply impressed the detail of digital holographic experiment and acquainted with the usage of the hardware concerned in optics-based technology, as well as computer programming language such as LabVIEW and Matlab.

\section{Basic principle of FINCH}

The scheme of the FINCH experimental setup is shown in Fig. 1. The object $\mathrm{O}$ (USAF 1951 resolution chart) is back illuminated by a lamp WS. The transmitted light is filtered to a quasi-monochromatic light using an interference filter $\mathrm{F}$ with $633 \mathrm{~nm}$ central wavelength and $10 \mathrm{~nm}$ bandwidth. A lens L is used to roughly collimate the beam. The two beams split by the SLM (Holoeye LC-R 2500, working under phase mode) can interference each other on the COMS camera plane after reflecting by the beam splitter BS. The corresponding hologram is recorded and stored in the computer. 


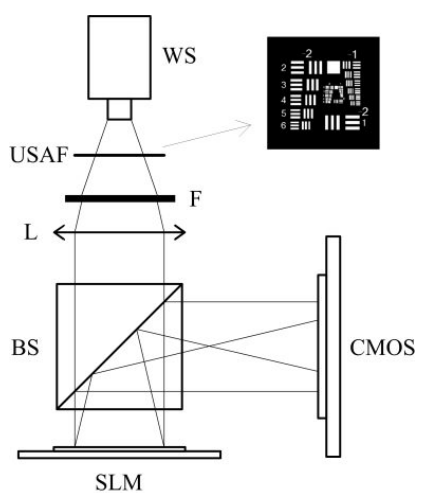

Fig. 1. Scheme of the FINCH experimental system. WS, white light lamp; USAF, object; F, interference filter; L, lens; BS, beam splitter; SLM, spatial light modulator; CMOS, digital camera.

The object is assumed consist of many spatially incoherent point light source. For each point, the emitted spherical wavefront is collimated by the lens $\mathrm{L}$ after filtered by the filter $F$. The resulting quasi-monochromatic light with central wavelength of $\lambda$ propagates to the SLM. The beam is split into a spherical wave and a plane wave after reflected by the SLM. The DOE displayed on the SLM has a transmittance function of

$$
P(x, y)=\frac{1}{2}+\frac{1}{2} \exp \left[-\frac{i \pi}{\lambda f_{d}}\left(x^{2}+y^{2}\right)+i \theta\right],
$$

where the first term of $P(x, y)$ indicates a plane mirror, and the second term indicates a positive lens with the focal length of $f_{d} . \theta$ is the phase shift values. Referring to the three-step phase shifting technique, $\theta_{1}=0, \theta_{2}=2 \pi / 3$ and $\theta_{3}=4 \pi / 3$ respectively. Three holograms $H_{1}, H_{2}$ and $H_{3}$ are recorded sequentially corresponding to the three phase shift values. Then the complex hologram without the zero order and twin image can be built as

$$
\begin{aligned}
H_{F}(x, y) & =H_{1}(x, y)\left[\exp \left(-i \theta_{3}\right)-\exp \left(-i \theta_{2}\right)\right]+H_{2}\left[\exp \left(-i \theta_{1}\right)-\exp \left(-i \theta_{3}\right)\right] \\
& +H_{3}(x, y)\left[\exp \left(-i \theta_{2}\right)-\exp \left(-i \theta_{1}\right)\right] .
\end{aligned}
$$

The object $U_{r}$ can be reconstructed digitally from $H_{F}$ using the Fresnel diffraction algorithm as

$$
U_{r}(x, y)=F F T^{-1}\left\{F F T\left[H_{F}(x, y)\right] \times \exp \left[-j \pi \lambda z_{r}\left(f_{x}^{2}+f_{y}^{2}\right)\right]\right\},
$$

where $F F T$ and $F F T^{1}$ indicate the fast Fourier transform and inverse fast Fourier transform, respectively. $z_{r}$ is the reconstruction distance, $f_{x}$ and $f_{y}$ indicate the spatial frequency coordinates. The structures located at any depth in the object volume can be focused by choosing a suitable $z_{r}$, thus 3D imaging can be achieved. The reconstructed intensity distribution of the object $I_{r}$ can be obtained as

$$
I_{r}(x, y)=\left|U_{r}(x, y)\right|^{2},
$$

while the phase profile can be also extracted as

$$
\phi_{r}(x, y)=\arctan \left\{\frac{\operatorname{Im}\left[U_{r}(x, y)\right]}{\operatorname{Re}\left[U_{r}(x, y)\right]}\right\} .
$$


It should be emphasized that the phase $\phi_{r}$ here is not the quantitative phase distribution of the object, because the object is spatially incoherent illuminated. During the recording of the hologram in FINCH, series of hardware such as the SLM and CMOS have to be operated together and synchronized. Some digital algorithms are also concerned in reconstruction. Thus a global control application is necessary for improving the operation speed and reducing the errors introduced by the mechanical shake of the system. As a kind of graphical programming platform, LabVIEW is of great suitable for the learning of students because it has a relative easy and readable user interface.

\section{Programming of the control application based on LabVIEW}

Before the design of specific functions, communications and data transmission between the hardware should be built in the application. In the system, the DOE displayed on the SLM is first generated in the computer and then sent to the SLM trough the graphic card. The generation and display of the DOE is achieved by invoking the Mathscript function and graphic data communication module of the LabVIEW. By invoking the functions in dynamic link library (DLL) file, action control, parameters setting and data acquisition of the CMOS can be implemented in LabVIEW environment through a specific image acquisition card. Other hardware such as the power meter is controlled through the corresponding DLL or ActiveX files.

The main functions of the application include the detection of the light power, generation of the DOE with different parameters, capturing and the reconstruction of the hologram. Among them, the generation of the DOE and reconstruction of the hologram are both achieved by invoking the Mathscript and programming in Matlab language. The design method of the application is illustrated using the example of DOE generation function. The transmittance function in Eq. (1) is generated by first build a zero matrix corresponding to the parameter of the SLM. Then the entire matrix is separated into two parts by random selecting half of the pixels. The values of one half of the pixels are chosen as the phase distribution of a positive lens with focal length of $f_{d}$, while the values for other half of the pixels is set to be constant. The corresponding original graphical program code is shown in Fig. 2. One of the generated DOE mask is shown in Fig. 3.

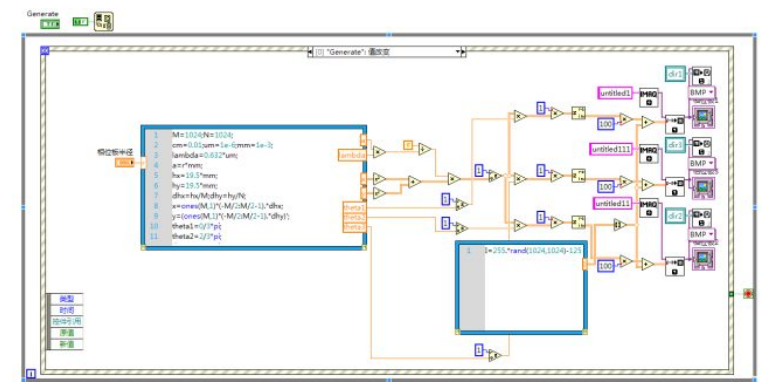

Fig. 2. Original graphical program code for DOE generation. 


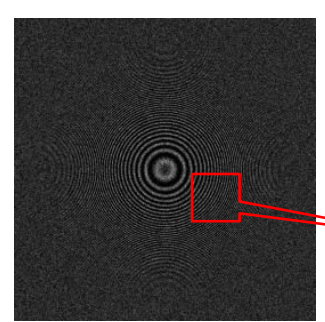

(a)

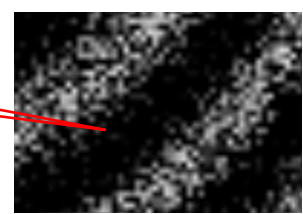

(b)

Fig. 3. One of the generated DOE mask (a) and the magnified display (b).

The global operation interface of the application is shown in Fig. 4. The layout of the interface is: the buttons at upper left corner refer to the capturing of the hologram and the generation of the DOE; the buttons at bottom left corner refer to the basic function of the power meter; the buttons and window at right refer to the real time display of the view of CMOS, reconstruction of the hologram and the display of the intensity and phase of the reconstructed image.

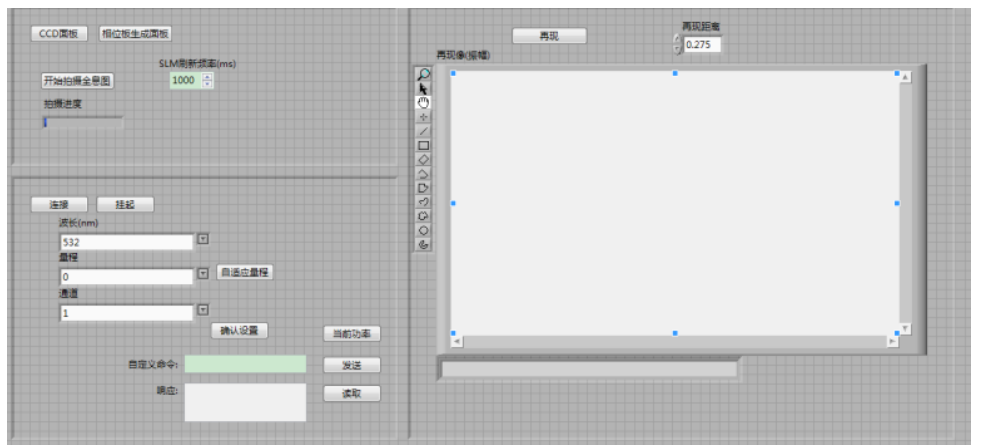

Fig. 4. The global operation interface of the application.

The flow chart for practical operation the FINCH experiment using the application can be concluded as:

1. Go to the DOE generation interface and input the desired parameters, three DOE will be generated and displayed blow after click the generation button;

2. Back to the global operation interface; adjust the position of the CMOS referring to the real time view at the right; three holograms will be recorded and saved to the given path after clicking the capture button;

3. Input the reconstructed distance, the reconstructed intensity and phase image will be displayed after clicking the reconstruction button at upper right of the main interface.

The experimental result using the application is shown in Fig. 5. Reconstructed intensity image is shown in the right of the interface, while the phase image is shown in the left window. 


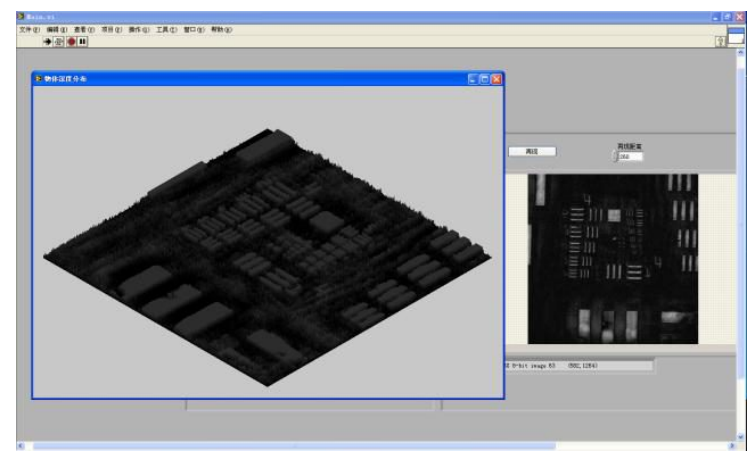

Fig. 5. The experimental result using the application

\section{Conclusions}

In this paper a control application of FINCH experiment is built on LabVIEW platform for automatic recording and reconstructing the digital hologram. The operation speed can be great improved using the application. At the same time, the noise and error introduced by the manual manipulation will be eliminated. The graphical programming method of LabVIEW makes the application more readable. The students are stimulated to practice the basic principle of the incoherent digital holography and some programming skills about the controlling of optics-based hardware. The training will help to improve the interest and better understanding the complex physical concept and phenomena in some courses such as Fourier optics or statistical optics.

\section{References}

[1] Y. Wan, S. Tao, Z. Jiang, D. Wang, and Y. Wang, "Active and interactive teaching based on exploring forefront topics in information optics," International Topical Meeting on Education \& Training in Optics an Photonics 2009, St. Asaph, North Wales, United Kingdom, 5-7 June, paper ESCB4 (2009).

[2] L. Mertz, and N. O. Young, "Fresnel transform of images," Proc. of the International Conference on Optical Instruments and Techniques, Chapman Hall, London, 305-310 (1961).

[3] J. Rosen, and G. Brooker, "Non-scanning motionless fluorescence three-dimensional holographic microscopy," Nat. Photon. 2(3), 190-195 (2008). 